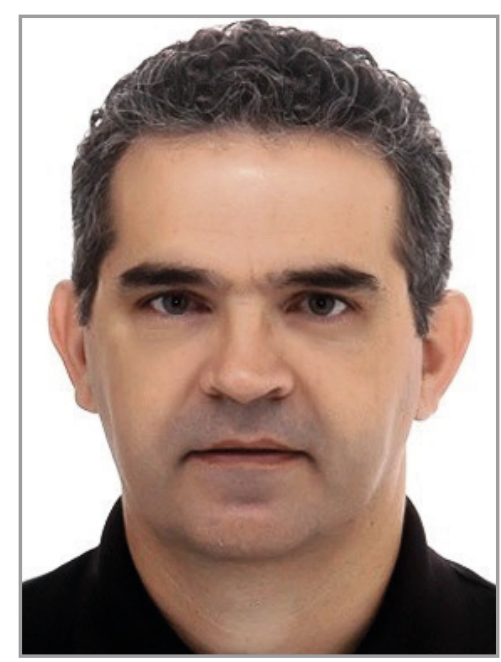

\section{Chromatographic Challenges}

\author{
Renato Zanella (1D) \\ Full Professor \\ Laboratory for Pesticide Residue Analysis (LARP) \\ Chromatography and Mass Spectrometry Research Group (CPCEM) \\ Department of Chemistry, Federal University of Santa Maria \\ Santa Maria, RS, Brazil
}

Chromatography, with its varied analytical possibilities, plays a prominent role in the various analyses, especially of organic compounds, and it has applications in several application areas, such as environmental, forensics, metabolomics, clinical, and industrial. Coupling chromatography with mass spectrometry has provided broad, fast, selective, and sensitive methods. Chromatography coupled to high-resolution or tandem mass spectrometry has enabled multiresidue and multi-class analyses with great performance for different complex matrices. The use of high-resolution mass spectrometry allows target, non-target, and suspect screening analyses of a large number of compounds, which is of great importance today. Comprehensive chromatography technologies are adequate tools for complex samples, such as petrochemicals, natural products, foods, and clinical applications. Innovations in chromatography columns and the higher performance of new equipment systems in recent decades have allowed for faster analyses without sacrificing the reliability of the results.

Chromatography allows for the quality control of food and beverages through the analysis of pesticide multiresidue, veterinary drugs, mycotoxins, dyes, preservatives, and contaminants contained in the packaging. This technique is also important in determining functional compounds, vitamins, and antioxidants, among other parameters, present in foods. These analyses ensure the authenticity of the products tested. In clinical analysis, chromatography has played a fundamental role in disease diagnosis and prevention, as well as in supporting clinical evaluations during and after complex surgeries. It can also be used to assess the exposure of workers to pollution by determining the pollutants found in ambient air or in biological fluids, such as blood and urine. In the environmental area, chromatography allows to evaluate the occurrence of persistent organic pollutants, as well as emerging pollutants in different matrices, such as in water, effluents, air, soil, and sediments. The analysis of contaminants in wild animals is of great importance and can be used to evaluate the exposure of animals to contaminants and to establish measures to mitigate contamination problems. Multi-residue pesticide determinations are required today, but analyses of endocrine disruptors, pharmaceuticals, personal care products (PPCPs), and flame retardants have been increasingly frequent.

The determination of a wide range of organic compounds of interest has required a great effort from the instrumentation companies in the development of sensitive and selective equipment in order to achieve the required levels. Despite this effort, the training of new analysts prepared to develop new analytical methods is a very important challenge. Continuous investment in the qualification of laboratories in terms of infrastructure and training of analysts is very important, including collaboration of the instrumentation companies and the laboratories that require these analysts. Cooperation between research groups can provide each with access to new technologies for more complex applications. 
The establishment and application of faster and more efficient analytical methods for a large number of compounds makes the implementation of a quality system, such as ISO 17025, even more important in enabling more efficient control of working conditions and traceability of results. The qualification of personnel with experience in sample preparation and analysis techniques, as well as in quality system, should be encouraged since the demand for professionals with these skills is increasing due to the need for reliable results in the most diverse sectors. In chromatography, speed and accuracy are always being pushed to the limits by the analytical demands of real samples. 